\title{
Dispersion Under Iteration of Strongly Mixing Transformations on Metric Spaces **
}

\author{
Hana Fatkić ${ }^{*}$ Abe Sklar ${ }^{+}$, Huse Fatkić ${ }^{\#}$ \\ Accepted 15 ${ }^{\text {th }}$ August 2014 \\ DOI: 10.18100/ijamec.90047
}

Abstract: In this paper, we investigate metric properties and dispersive effects of strongly mixing transformations on general metric spaces endowed with a probability measure; in particular, we investigate their connections with the theory of generalized ( $\alpha$-harmonic) diameters on general metric spaces. We first show that the known result by R. E. Rice ([Aequationes Math. 17(1978), 104-108], Theorem 2) (motivated by some physical phenomena and offer some clarifications of these phenomena), which is a substantial improvement of Theorems 1 and 2 due to T. Erber, B. Schweizer and A. Sklar [Comm. Math. Phys., 29 (1973), 311 - 317], can be generalized in such a way that this result remains valid when "ordinary diameter" is replaced by " $\alpha$-harmonic diameter of any finite order". Next we show that "ordinary essential diameter" in the mentioned Rice's result can be replaced by the" essential $\alpha$-harmonic diameter of any finite order". These results also complement the previous results (on dynamical systems with discrete time and/or generalised diameters) of N. Faried and M. Fathey, H. Fatkić, E. B. Saff, S. Sekulović and V. Zakharyuta.

Keywords: Dispersion under iteration, diameter, harmonic n-diameter, measurability-preserving dynamical systems, measure, metric space, mixing transformations, probability space.

\section{Introduction}

In this work we shall study actions of the group $\boldsymbol{Z}$ of integers on a probability space $X$, i.e., we study a transformation $T: X \rightarrow X$ and its iterates $\boldsymbol{T}^{\boldsymbol{n}}, n \in \boldsymbol{Z}$.

Specifically, we shall investigate some metric properties and dispersive effects of strongly mixing (SM) transformations on general metric spaces endowed with a probability measure; in particular, we shall study connections of SM discrete time dynamical systems with the theory of generalized diameters. We shall generally refer to Billingsley [1], Cornfeld, Fomin and Sinai [2], Fatkić [5], Hille [10], Schweizer and Sklar [14].

Suppose $(X, \mathcal{A}, P)$ is a probability space. As usual, a transformation $T: X \rightarrow X$ is called:

(i) measurable $(P$ - measurable) if, for any $A$ in $\mathcal{A}$, the inverse image $T^{-1}(A)$ is in $\mathcal{A}$;

(ii) measure-preserving if $T$ is measurable and $P\left(T^{-1}(A)\right)=$ $P(A)$ for any $A$ in $\mathcal{A}$ (or, equivalently, measure $P$ is said to be invariant under $T$ );

(iii) ergodic if the only members $A$ of $\mathcal{A}$ with $T^{-1}(A)=A$ satisfy $P(A)=0$ or $P(X \backslash A)=0$;

(iv) weakly mixing (or weak-mixing) (with respect to $P$ ) if $T$ is

"Department for Computer Science and Informatics, Faculty of Electrical Engineering, University of Sarajevo, Zmaja od Bosne bb, Sarajevo, BiH 71000, Bosnia and Herzegovina; RFE/RL, Vinohradska 159A, Prague 10 CZ 100 00, Czech Republic, fatkic.hanna@ gmail.com

${ }^{+}$Department of Mathematics, Illinois Institute of Technology, Chicago, IL 60616, USA

"Department of Mathematics, Faculty of Electrical Engineering,

University of Sarajevo, Zmaja od Bosne bb, Sarajevo, BiH 71000, Bosnia and Herzegovina,hfatkic@etf.unsa.ba

** This paper has been presented at the International Conference on Advanced Technology\&Sciences (ICAT'14) held in Antalya (Turkey), August 12-15, 2014.
$P$-measurable and

$$
\lim _{n \rightarrow \infty} \frac{1}{n} \sum_{i=0}^{n-1}\left|P\left(T^{-i}(A) \cap B\right)-P(A) P(B)\right|=0
$$

for any two $P$ - measurable subsets $A, B$ of $X$;

(v) strongly mixing (or mixing, strong-mixing) (with respect to $P$ ) if $T$ is $P$ - measurable and

$$
\lim _{n \rightarrow \infty} P\left(T^{-n}(A) \cap B\right)=P(A) P(B)
$$

for any two $P$-measurable subsets $A, B$ of $X$.

We say that the transformation $T: X \rightarrow X$ is invertible if $T$ is one-to-one (monic) and such that $T(A)$ is $P$ - measurable whenever $A$ is $P$-measurable subset of $X$.

A transformation $T$ on a probability space $(X, \mathcal{A}, \mu)$ is said to be measurability - preserving if $T(\mathcal{A}) \subseteq \mathcal{A}$ (i. e., if $T(A)$ is $P$ measurable whenever $\mathrm{A}$ is $\mathrm{P}$ - measurable). In this case we also say that the transformation $T$ preserves $P$ - measurability.

If ( $X, \mathcal{A}, P)$ is a probability space, and $T: X \rightarrow X$ is a measurepreserving transformation (with respect to $P$ ), then we say that $\Phi$ $:=(X, \mathcal{A}, P, T)$ is an abstract dynamical system. An abstract dynamical system is often called a dynamical system with discrete time or a measure-theoretic dynamical system or an endomorphism. We shall say that the abstract dynamical system $\Phi$ is: (i) invertible if $T$ is invertible; (ii) ergodic if $T$ is ergodic; (iii) weakly (resp. strongly) mixing if $T$ is weakly (resp. strongly) mixing (see [2, pp. 6-26]).

If $T$ is a strongly mixing transformation of a probability space ( $X$, $\mathcal{A}, P$ ), then, as is well-known, $T$ is both measure-preserving and ergodic. Furthermore, if $T: X \rightarrow X$, in addition (to being strongly 
mixing on $X$ with respect to $P$ ), is invertible, then (1.1) is equivalent to (the well- known result):

$$
\lim _{n \rightarrow \infty} P\left(T^{n}(A) \cap B\right)=P(A) P(B)
$$

for any $P$-measurable subsets $A, B$ of $X$.

In this work we consider a metric space/(extended metric spaces, i.e., if we allow $d$ to take values in $[0, \infty]$ (the nonnegative lower reals) instead of just in $[0, \infty)$, then we get extended metric spaces) $(X, d)$ on which a probability measure $P$ is defined. The domain of $P$, a $\sigma$ - algebra $\mathcal{A}$ of subsets of $X$, is assumed to include all Borel sets in $(X, d)$; in particular, therefore, all open balls in $(X, d)$ are $P$ - measurable. We call $P$ nonsingular if there exist two open balls $K_{1}, K_{2}$, and a positive number $x_{0}$ such that $P\left(K_{1}\right)>0, P\left(K_{2}\right)>$ 0 , and $d\left(w_{1}, w_{2}\right)>0$ for all $w_{1}$ in $K_{1}, w_{2}$ in $K_{2}$. We call $P$ pervasive if $P(K)>0$ for all open balls $K$ in $X$. Note that any pervasive measure is nonsingular if $X$ has more than one point.

The (ordinary) diameter of a subset $A$ of $X$, i.e., the supremum of the set $\{d(x, y) \mid x, y \in A\}$, will be denoted by diam $(A)$. We can define the diameter of the empty set (the case $A=\varnothing$ ) as 0 or $-\infty$, as we like. But, like many other authors, we prefer to treat the empty set as a special case, assigning it a diameter equal to 0 , i.e., diam $(\varnothing)=0$, which corresponds to taking the codomain of $d$ to be the set of all nonnegative real numbers (if the distance function $d$ is viewed here as having codomain $\mathbf{R}$, this implies that $\operatorname{diam}(\varnothing)=-\infty)($ see [5] and [10]).

If $A$ is $P$-measurable, then the (ordinary) essential diameter of $A$, denoted by ess diam $(A)$, is the infimum of the set of diameters of all $P$-measurable sets $B$ such that $B \subseteq A$ and $P(B)=P(A)$.

Let $(X, d)$ be a metric space/(extended metric space), and let $A$ be a subset of $X$. For any positive integer $k \geq 2$ and $\alpha>0$, we define the $\alpha$-harmonic diameter of order $k$ of $A$ ( $k$ th $\alpha$ harmonic diameter of $A$ ), denoted by $D_{k}^{(\alpha)}(A)$, to be the quantity

$$
D_{k}^{(\alpha)}(A):=\sup \left\{\left(\begin{array}{c}
k \\
2
\end{array}\right)\left(\sum_{1 \leq i<j \leq k}\left[d\left(x_{i}, x_{j}\right)\right]^{-\alpha}\right)^{-1} \mid x_{1}, \ldots, x_{k} \in A\right\} .
$$

Note that $D_{2}^{(1)}(A)$ is the (ordinary) diameter (diam $\left.(A)\right)$ of the set $A$. The sequence $\left(D_{k}^{(\alpha)}(A)\right)$ can be shown to be monotone nonincreasing (see, e.g., [5], [8], [10] and [11]), and therefore has a limit as $k$ tends to infinity. By definition, the $\alpha$-harmonic transfinite diameter (the $\alpha$-logarithmic capacity) of $A$ is

$$
\tau^{(\alpha)}(A)(=\operatorname{cap}(A)):=\lim _{k \rightarrow \infty} D_{k}^{(\alpha)}(A) .
$$

Note that $0 \leq \tau^{(\alpha)}(A) \leq D_{2}^{(\alpha)}(A) \leq \operatorname{diam}(A)$, and that

$$
B \subseteq A \text { implies } \tau^{(\alpha)}(B) \leq \tau^{(\alpha)}(A) \text {. }
$$

If $A$ is $\mu$-measurable subset of $X$, then, for any positive integer $k$ $\geq 2$, the essential $\alpha$-harmonic diameter of order $k$ of $A$, denoted by ess $D_{k}^{(\alpha)}(A)$, is the infimum of the set of $\alpha$-harmonic diameters of all $\mu$-measurable sets $B$ such that $B \subseteq A$ and $\mu(B)=$ $\mu(A)$, i.e.,

$$
\text { ess } D_{k}^{(\alpha)}(A):=\inf \left\{D_{k}^{(\alpha)}(B): B \in \mathcal{A}, B \subseteq A, \mu(B)=\mu(A)\right\} \text {. }
$$

If $A$ consists of only finitely many points, then $\tau^{(\alpha)}(A)=0$. The general theory of generalised diameters and transfinite diameters plays an important role in complex analysis. It is related to the logarithmic potential theory with applications to approximation theory and the Čebyšev constant (see, e.g., [4], [10], [13] and [16]).

In the classical case, when $\alpha=1, X=\mathbf{R}^{\mathbf{3}}, d$ the Euclidean metric on $\mathbf{R}^{3}$, and $A$ is a compact subset of $X$, the value $D_{k}^{(\alpha)}(A)(k \in$ $(\mathbf{N} \backslash\{1\})$ ), which is now called the harmonic diameter of the order $k$ of the set $A$, has a simple physical interpretation. In fact, if we look the distribution in $A$ of $k$ point sources of electric charge of size $1 / \mathrm{k}$, then the minimum potential energy of such a system of charges obtained when these charges are in the points $q_{i}^{(k)}(i=1, \ldots, k)$ where the function $f$, defined by

$$
f\left(x_{1}, \ldots, x_{k}\right):=\left(\begin{array}{l}
k \\
2
\end{array}\right)^{-1} \sum_{1 \leq i<j \leq k}\left[d\left(x_{i}, x_{j}\right)\right]^{-1},
$$

achieves a minimum value. In addition, the value of the potential energy is

$$
\frac{k-1}{2 k} \cdot \frac{1}{D_{k}^{(1)}(A)} .
$$

Any set of $k$ points that attains this minimal energy is called an equilibrium configuration for $A$.

Investigations in ([3], [5] - [9], [12] and [14]) have shown, however, that many important consequences of (1.2) persist in the absence of invertibility and/or the strongly mixing property. The following results (the most useful results of these investigations for the goals of this paper) is due to R.E. Rice [17, Theorems 1 and 2]:

Theorem 1.1. Let $T$ be a strongly mixing transformation on the probability space $(X, \mathcal{A}, P)$. If $T$ is measurability - preserving, then for any $P$-measurable subsets $A, B$ of $X$,

$$
\lim _{n \rightarrow \infty} \mu\left(T^{n}(A) \cap B\right)=\mu(B) \lim _{n \rightarrow \infty} \mu\left(T^{n}(A)\right) .
$$

Theorem 1.2. Let $(X, d)$ be a metric space, let $\mathcal{A}$ be a $\sigma$-algebra of subsets of $X$ and $P$ a probability measure on $\mathcal{A}$. Suppose further that every open ball in $(X, d)$ is P-measurable and has positive measure. Let $T$ be a transformation on $X$ that is strongly mixing with respect to $P$ and suppose that $A$ is $P$-measurable subset of $X$ with positive measure. Then

(i) $\quad \lim _{n \rightarrow \infty} \operatorname{diam}\left(T^{n}(A)\right)=\operatorname{diam}(X)$.

(ii) If in addition, $T$ is forward measurable, i.e., if $T(B)$ is $P$ measurable whenever $B$ is $P$ - measurable, then $\lim _{n \rightarrow \infty} \operatorname{ess} \operatorname{diam}\left(T^{n}(A)\right)=\operatorname{diam}(X)$.

Theorems 1.1. and 1.2 have many consequences which are of interest because of the extreme simplicity of both their mathematical and physical realizations. Among others, these consequences have great relevance in the discussion of the recurrence paradox of Statistical Mechanics (see the previous results of T. Erber, B. Schweizer and A. Sklar [3], B. Schweizer and A. Sklar [14, pp. 181-190 and (in Dover Edition) 295-297] and H. Fatkić ([5] - [7]). It is therefore interesting to investigate how the conclusions of Theorem 1.2 must be modified when the the ordinary diameter is replaced by the $\alpha$-harmonic diameter of any finite order.

\section{Main results}

Strongly mixing transformations exhibit dispersive effects. Thus, for example, he dispersive character of the functions $C_{n}$ defined by

$$
C_{n}(x)=2 \cos \left(n \arccos \left(\frac{x}{2}\right)\right)\left(=2 \check{\mathrm{C}}_{n}\left(\frac{x}{2}\right)\right),
$$

where $\check{C}_{n}$ is the standard $n$th - degree Čebyšev polynomial) is brought out by the fact that if $I$ is a subinterval of $[-2,2]$ with $P_{c}(I)>0$ (where $P_{c}$ is the Lebesgue - Stieltjes $F_{\mathbf{c}}$ measure on $[-2,2]$ determined by

$$
F_{c}(x)=\frac{1}{2}+\left(\frac{1}{\pi}\right) \arcsin \left(\frac{x}{2}\right)
$$

if $n \geq 2$ and $m$ is any integer such that $m \geq\left(\log \left(2 / P_{c}(I)\right)\right) / \log n+2$,

then $C_{n}^{m}(I)=[-2,2]$; i.e. , the $m$ th image of $I$ is the entire space (see [14, p.184] and [5]). 
A strongly mixing transformation $T$ that is one to one cannot exhibit such extreme behavior, for in this case $T^{-1}$ is also measure preserving. Nevertheless, as was shown in [3] and [12], under iteration, all strongly mixing transformations tend to spread sets (of positive measure) out in ordinary diameter.

Our aim in this work is to show that the known R. E. Rice's Theorem 1.2 [12, Theorem 2] (motivated by some physical phenomena and offer some clarifications of these phenomena) can be generalized in such a way that (1.7) remains valid when "(ordinary) diameter" is replaced by " $\alpha$-harmonic diameter of any finite order" and that (1.8) remains valid when "(ordinary) essential diameter" is replaced by "essential $\alpha$-harmonic diameter of any finite order". These results also complement the previous results of N. Faried and M. Fathey [4], H. Fatkić ([5] and [7]), Huse Fatkić, S. Sekulović, and Hana Fatkić ([8] and [9]), E. B. Saff [13] and V. Zakharyuta [16].

We first show that (1.7) holds, not only for the diameter, but also for the $\alpha$-harmonic diameter $D_{k}^{(\alpha)}(A)$ of order $k$ which is defined for any positive integer $k \geq 2$ and any $A \subseteq X$ by (1.3). Next we show that essential diameter in (1.8) can be replaced by the essential $\alpha$-harmonic diameter ess $D_{k}^{(\alpha)}(A)$ of order $k$ which is defined for any positive integer $k \geq 2$ and any $A \subseteq X$ by (1.5). But here the requirement that transformation $T$ be measurability -preserving is necessary because the essential $\alpha$-harmonic diameter is defined only for measurable sets. All of these results have some importance, not only in Mathematics, but also in Statistical Mechanics.

Note that measurability-preserving strongly mixing transformations on a finite measure space are generalizations of the invertible strongly mixing transformations (they are not necessarily one-to-one).

Lemma 2.1. Let $(X, \mathcal{A}, P)$ be a probability space. Let $T$ be a transformation on $X$ that is strongly mixing with respect to $P$ and suppose that $B_{1}, B_{2}, \ldots, B_{k}(k \in \mathbf{N})$ are P-measurable subsets of $X$. If $P(A)>0$ and $P\left(B_{i}\right)>0 \quad(i=1, \ldots, k)$, then there is a positive integer $n_{0}=n_{0}(k)$ such that

$$
T^{n}(A) \cap B_{i} \neq \varnothing
$$

for $i=1,2, \ldots, k$ and all $n>n_{0}$.

Proof. Since $T$ is strongly mixing and $P(A) P\left(B_{i}\right)>0$ for all $i \in$ $\{1, \ldots, k\}$, it follows that there is a positive integer $n_{0}\left(n_{0}=n_{0}(k)\right)$ such that

$$
\mu\left(A \cap T^{-n}\left(B_{i}\right)\right)>0,
$$

for $i=1,2, \ldots, k$ and all $n>n_{0}$,whence

$$
A \cap T^{-n}\left(B_{i}\right) \neq \varnothing
$$

for $i=1,2, \ldots, k$ and all $n>n_{0}$. Since

$$
\begin{aligned}
T^{-n}\left(T^{n}(A) \cap B_{i}\right) & =\left(T^{-n}\left(T^{n} A\right)\right) \cap T^{-n}\left(B_{i}\right) \\
& \supseteq A \cap T^{-n}\left(B_{i}\right)
\end{aligned}
$$

for all $i=1,2, \ldots, k$ and every $n$ in $\mathbf{N}, n>n_{0}$, it follows, by (2.2), that $T^{-n}\left(T^{n}(A) \cap B_{i}\right) \neq \varnothing$ and therefore (2.1) holds and the proof of Lemma 2.1 is complete.

Theorem 2.1. Let $(X, d)$ be a metric space, let $\mathcal{A}$ be a $\sigma$ algebra of subsets of $X$ and $P$ a probability measure on $\mathcal{A}$. Suppose further that every open ball in $(X, d)$ is P-measurable and has positive measure. Let $T$ be a transformation on $X$ that is strongly mixing with respect to $P$ and suppose that $A$ is $P$ measurable subset of $X$ with positive measure. Then

$$
\lim _{n \rightarrow \infty} D_{k}^{(\alpha)}\left(T^{n}(A)\right)=D_{k}^{(\alpha)}(X),
$$

where $D_{k}^{(\alpha)}(A)$, is the $\alpha$-harmonic diameter of order $k$ given by (1.3).

Proof. Throughout this proof we will denote $D_{2}^{(\alpha)}(X)$, the $\alpha$ diameter of $X$, by $D$, and $\sum_{1 \leq i<j \leq k} a_{i j} \quad$ by $\quad \sum_{k} a_{i j}$.

Let $k$ be an arbitrary positive integer greater than 1 . Since $D_{k}^{(\alpha)}$ is a monotone nondecreasing set function it is clear that (2.4) holds when $D_{k}^{(\alpha)}(X)=0$.

Suppose next that $0<D_{k}^{(\alpha)}(X)<+\infty$, whence also $0<D<$ $+\infty$, and let $\varepsilon>0$ be given. Then, by (1.3), there exist points $x_{1}, x_{2}, \ldots, x_{k}$ in $X$ such that

$$
\left(\begin{array}{l}
k \\
2
\end{array}\right)\left\{\sum_{k}\left[d\left(x_{i}, x_{j}\right)\right]^{-\alpha}\right\}^{-1}>D_{k}^{(\alpha)}(X)-\frac{\varepsilon}{2}
$$

Let $m$ be a positive integer and let $B_{1} B_{2} \ldots, B_{\mathrm{k}}$ be open balls of radius $1 / 2 \mathrm{~m}$ centered at $x_{1}, x_{2}, \ldots, x_{k}$, respectively.

Next, since $T: X \rightarrow X$ is strongly mixing with respect to $P$ and since $P(A)>0$ and $P\left(B_{i}\right)>0$ for all $i=1,2, \ldots, k$, it follows from Lemma 2.1 that there is a positive integer $n_{0}\left(n_{0}=n_{0}(k, m)\right)$ such that $T^{n}(A) \cap B_{i} \neq \varnothing$ for $i=1,2, \ldots, k$ and all $n>n_{0}$. Given $n \geq n_{0}$, for each $i=1,2, \ldots, \mathrm{k}$, choose a point $y_{i}$ in $T^{n}(A) \cap B_{i}$. Then, for all $i, j=1, \ldots, k$,

$d\left(x_{i}, x_{j}\right) \leq d\left(x_{i}, y_{i}\right)+d\left(y_{i}, y_{j}\right)+d\left(y_{j}, x_{j}\right)<d\left(y_{i}, y_{j}\right)+\frac{1}{m}$, whence, by (2.5), we have

$$
\begin{aligned}
D_{k}^{(\alpha)}(X)-\frac{\varepsilon}{2} & <\left(\begin{array}{l}
k \\
2
\end{array}\right)\left\{\sum_{k}\left[d\left(x_{i}, x_{j}\right)\right]^{-\alpha}\right\}^{-1} \\
& <\left(\begin{array}{c}
k \\
2
\end{array}\right)\left\{\sum_{k}\left[d\left(y_{i}, y_{j}\right)+\frac{1}{m}\right]^{-\alpha}\right\}^{-1} .
\end{aligned}
$$

Since

$$
\left(\begin{array}{l}
k \\
2
\end{array}\right)\left\{\sum_{k}\left[d\left(y_{i}, y_{j}\right)\right]^{-\alpha}\right\}^{-1} \leq D_{k}^{(\alpha)}\left(T^{n}(A)\right)
$$

and

$$
\begin{gathered}
\left(\begin{array}{l}
k \\
2
\end{array}\right)\left\{\sum_{k}\left[d\left(y_{i}, y_{j}\right)+\frac{1}{m}\right]^{-\alpha}\right\}^{-1}=\left(\begin{array}{l}
k \\
2
\end{array}\right)\left\{\sum_{k}\left[d\left(y_{i}, y_{j}\right)\right]^{-\alpha}\right\}^{-1} \\
+\left(\begin{array}{c}
k \\
2
\end{array}\right)\left\{\left\{\sum_{k}\left[d\left(y_{i}, y_{j}\right)+\frac{1}{m}\right]^{-\alpha}\right\}^{-1}-\left\{\sum_{k}\left[d\left(y_{i}, y_{j}\right)\right]^{-\alpha}\right\}^{-1}\right\} \\
\leq\left(\begin{array}{c}
k \\
2
\end{array}\right)\left\{\sum_{k}\left[d\left(y_{i}, y_{j}\right)\right]^{-\alpha}\right\}^{-1}+\Delta(k, m),
\end{gathered}
$$

where $\Delta(k, m)$ is the positive value such that $\lim _{m \rightarrow \infty} \Delta(k, m)=$ 0 , we have

$$
D_{k}^{(\alpha)}\left(T^{n}(A)\right)+\Delta(k, m)>D_{k}^{(\alpha)}(x)-\frac{\varepsilon}{2} .
$$

Hence, and from the fact that there is a positive integer $m$ such that $\Delta(k, m)<\varepsilon / 2$ ( $k$ fixed) for all $m \geq m_{0}$, it follows that

$$
D_{k}^{(\alpha)}\left(T^{n}(A)\right)>D_{k}^{(\alpha)}(X)-\varepsilon
$$

holds for every $n \geq N$, and every $\varepsilon>0$, whence

$$
\liminf _{n \rightarrow \infty} D_{k}^{(\alpha)}\left(T^{n}(A)\right) \geq D_{k}^{(\alpha)}(X) \text {. }
$$

But, clearly, $\lim _{n \rightarrow \infty} \sup _{n \rightarrow \infty} D_{k}^{(\alpha)}\left(T^{n}(A)\right) \leq D_{k}^{(\alpha)}(X)$, whence we obtain (2.4).

Lastly, the case $D_{k}^{(\alpha)}(X)=+\infty$ can be treated by choosing for each positive integer $S$ a $k$-tuple of points $x_{1}, x_{2}, \ldots, x_{k}$ in $X$ such that

$$
\left(\begin{array}{l}
k \\
2
\end{array}\right)\left\{\sum_{k}\left[d\left(x_{i}, x_{j}\right)\right]^{-\alpha}\right\}^{-1}>s
$$

and then repeating the previous argument. This completes the proof of Theorem 2.1. 
Theorem 2.1 shows that any set $A$ of positive measure necessarily spreads out, not only in (ordinary) diameter, but also in $\alpha$ harmonic diameter of any finite order. Thus, even though $A$ may not spread out in "volume" (measure), there is a very definite sense in which $A$ does not remain small.

Corollary 2.1. Under hypotheses of Theorem 2.1, for any $P$ measurable subset $A$ of $X$ of positive measure,

$$
\lim _{k \rightarrow \infty}\left(\lim _{n \rightarrow \infty} D_{k}^{(\alpha)}\left(T^{n}(A)\right)\right)=\tau^{(\alpha)}(X),
$$

where $D_{k}^{(\alpha)}$ is the $\alpha$-harmonic diameter of order $k$ given by (1.3), and $\tau^{(\alpha)}$ is the $\alpha$-harmonic transfinite diameter given by (1.4).

Proof. The property (2.6) follows immediately from (2.4) and the fact that $\lim _{k \rightarrow \infty} D_{k}^{(\alpha)}=\tau^{(\alpha)}(X)$.

Theorem 2.2. Let $(X, d)$ be a metric space and $(X, \mathcal{A}, P)$ a finite measure space with the property that every open ball in $(X, d)$ is $P$-measurable and has positive measure. Let $(X, \mathcal{A}, P, T)$ be a measurability-preserving strongly mixing dynamical system with discrete time. Then, for any P-measurable subset A of $X$ of positive measure, and any positive integer $k \geq 2$,

$$
\lim _{k \rightarrow \infty} \operatorname{ess} D_{k}^{(\alpha)}\left(T^{n}(A)\right)=D_{k}^{(\alpha)}(X),
$$

where $D_{k}^{(\alpha)}$ is the $\alpha$-harmonic diameter of order $k$ given by (1.3), and $\operatorname{ess} D_{k}^{(\alpha)}$ is the essential $\alpha$-harmonic diameter of order $k$ which is defined for any any positive integer $k(k \geq 2)$ and any $\mu$ measurable subset $A$ of $X$ by (1.5).

Proof. Throughout this proof we will denote $D_{2}^{(\alpha)}(X)$, the $\alpha$ diameter of $X$, by $D$, and $\sum_{1 \leq i<j \leq k} a_{i j}$ by $\sum_{k} a_{i j}$

Note that the hypotheses of Theorem 2.2 are such that ess $D_{k}^{(\alpha)}(X)=D_{k}^{(\alpha)}(X)$. Let $k$ be an arbitrary positive integer greater than 1 . Since $D_{k}^{(\alpha)}$ is a monotone nondecreasing set function it is clear that (2.7) holds when $D_{k}^{(\alpha)}(X)=0$. To obtain the result it suffices to prove that (2.7) holds for

$$
0<D_{k}^{(\alpha)}(X)<+\infty .
$$

Suppose next that $0<D_{k}^{(\alpha)}(X)<+\infty$, whence also $0<D<$ $+\infty$, and let $\varepsilon>0$ be given. Then, by (1.3), there exist points $x_{1}, x_{2}, \ldots, x_{k}$ in $X$ such that $(2.5)$ holds.

Let $m(m=m(k, \varepsilon))$ be a positive integer and let $B_{1}, B_{2}, \ldots, B_{\mathrm{k}}$ be disjoint open balls of radius $1 / 2 m$ centered at $x_{1}, x_{2}, \ldots, x_{k}$, respectively.

Since $T: X \rightarrow X$ is measurability-preserving strongly mixing transformations with respect to $P$, it follows from (2.6) that there is a positive integer $N=N(k, m)$ such that

$$
P\left(T^{n}(A) \cap B_{i}\right)>0
$$

for $i=1,2, \ldots, k$ and all $n \geq N$. Let $n(n \geq N)$ be given. Let

$$
B=T^{n}(A) \cap\left(\bigcup_{i=1}^{k} B_{i}\right)
$$

For every $P$-measurable set $C \subseteq B$ such that $P(C)=P(B)$ we have $P\left(C \cap B_{i}\right)>0$ for $i=1,2, \ldots, k$, whence $C \cap B_{i} \neq \varnothing$ for $i=$ $1,2, \ldots, k$, and, arguing as in the proof of Theorem 2.1, we obtain $D_{k}^{(\alpha)}(C)>D_{k}^{(\alpha)}(X)-\varepsilon$. It follows that

$$
\begin{aligned}
\operatorname{ess} & D_{k}^{(\alpha)}\left(T^{n}(A)\right) \geq \operatorname{ess} D_{k}^{(\alpha)}(B) \\
\quad & \inf \left\{\operatorname{ess} D_{k}^{(\alpha)}(C): C \subseteq B, P(C)=P(B)\right\} \\
& \geq D_{k}^{(\alpha)}(X)-\varepsilon
\end{aligned}
$$

for every $\varepsilon>0$, and for all positive integers $n(n \geq N)$, whence

But, clearly,

$$
\liminf _{n \rightarrow \infty}\left[\operatorname{ess} D_{k}^{(\alpha)}\left(T^{n}(A)\right)\right] \geq D_{k}^{(\alpha)}(X) \text {. }
$$

$$
\limsup _{n \rightarrow \infty}\left[\operatorname{ess} D_{k}^{(\alpha)}\left(T^{n}(A)\right)\right] \leq D_{k}^{(\alpha)}(X)
$$

for every positive integer $n$, whence we obtain (2.7). This proves Theorem 2.2.

\section{Conclusion}

There is considerable evidence (see the proof of Theorem 2.1 and 2.2) to support a conjecture that our results (for strongly mixing dynamical systems with discrete time) which are contained in Theorems 2.1 and 2.2 can be extended to strongly mixing dynamical systems with continuous time (see [2, pp. 6-26]).

Even though a large set cannot have a small diameter and a set with a large diameter cannot be truly small, it is still true that the diameter of a set need not be a good measure of its size and shape. A better measure is furnished by the generalized diameters (see [14, pp. 181-190 and (in Dover Edition) 295-297] and also [5], [8], [10], [11] - [13] and [16]).

B. Schweizer and A. Sklar have been concluded that comparison of (11. 6.1) with (11.3.5) in [14] and the proof of the Rice's Theorem 1.2 quickly leads to the following conjecture [14, Problem 11.6.5]:

Problem 3.1. (Schweizer and Sklar, 1983) Does Theorem 1.2 remain valid when "diameter" is replaced by "(geometric) transfinite diameter"?

Note that our Theorems 2.1 and 2.2 in this paper is a first step toward the resolution of the above Problem 3.1. Also note that this problem could be formulated in a more general setting, i.e., not only for the compact set, but also for any set (with positive measure ) in a general metric space.

The transfinite diameter of a compact subset $A$ of a metric space is closely related, and often equal, to the capacity, and also to the Chebyshev constant. By the Fundamental Theorem of Classical Potential Theory, we have that, for any compact set $A$ in the complex plane,

$$
\operatorname{cap}(A)=\tau(A)=\text { čeb }(A)
$$

(see, e. g., [13]). Thus we may also pose the following conjecture (open problem):

Problem 3.2. Does the Rice's Theorem 1.2 remain valid when "diameter" is replaced by " $\alpha$-harmonic transfinite diameter"?

\section{References}

[1] P. Billingsley, Ergodic theory and information, John Wiley \& Sons, Inc., New York -London-Sydney, 1965.

[2] P. Cornfeld, S. V. Fomin,YA. G. Sinai, Ergodic Theory, Springer Verlag, NewYork-Heidelberg-Berlin, 1982.

[3] T. Erber, B. Schweizer, A. Sklar, Mixing transformations on metric spaces, Comm. Math. Phys. 29 (1973), 311 317.

[4] N. Faried, M. Fathey, " $S$-nuclearity and $n$-diameters of infinite Cartesian products of bounded subsets in Banach spaces", Acta Comment. Univ. Tartu. Math. 7 (2003), 4555.

[5] H. Fatkić, On probabilistic metric spaces and ergodic transformations, Ph. D. dissertation, Univ. Sarajevo, 2000, 170 pp; Rad. Mat. 10 (2) (2001), 261 - 263.

[6] H. Fatkić, "Characterizations of measurability-preserving ergodic transformations", Sarajevo J. Math. 1 (13) (2005), $49-58$.

[7] H. Fatkić, "Measurability-preserving weakly mixing transformations", Sarajevo J. Math. 2 (15) (2006), 159 172.

[8] H. Fatkić, S. Sekulović, and Hana Fatkić, "Further results on the ergodic transformations that are both strongly mixing and measurability preserving ", The sixth BosnianHerzegovinian Mathematical Conference, International 
University of Sarajevo, June 17, 2011, Sarajevo, B\&H; in: Resume of the Bosnian-Herzegovinian mathematical conference, Sarajevo J. Math. (20) (2011), 310 - 311.

[9] H. Fatkić, S. Sekulović, and Hana Fatkić, "Probabilistic metric spaces determined by weakly mixing transformations", in: Proceedings of the 2nd Mathematical Conference of Republic of Srpska-Section of Applied Mathematics, June 8\&9, 2012, Trebinje, B\&H, pp. 195208.

[10] E. Hille, Topics in classical analysis, in: Lectures on Mathematics (T. L. Saaty, ed.), Vol. 3, Wiley, New York, 1965, pp. 1-57.

[11] N. S. Landkof, Foundations of modern potential theory. Translated from the Russian by A. P. Doohovskoy. Die Grundlehren der mathematischen Wissenschaften, Band 180. Springer-Verlag, New York-Heidelberg, 1972.

[12] R. E. Rice, "On mixing transformations", Aequationes Math. 17 (1978), $104-108$.

[13] E. B. Saff, "Logarithmic potential theory with applications to approximation theory", Surv. Approx. Theory 5 (2010), $165-200$.

[14] Schweizer, A. Sklar, Probabilistic metric spaces, NorthHolland Ser. Probab. Appl. Math., North-Holland, New York, 1983; second edition, Dover, Mineola, NY, 2005.

[15] S. V. Tikhonov, "Homogeneous spectrum and mixing transformations", (Russian) Dokl. Akad. Nauk 436 (4) (2011), 448 - 451; translation in Dokl. Math. 83 (2011), 80 $-83$.

[16] V. Zakharyuta, "Transfinite diameter, Chebyshev constants, and capacities in $\boldsymbol{C}^{n}$ “. Ann Polon. Math. 106 (2012), 293313. 